\title{
Adult suicides linked to popular antidepressant
}

A drug that has been associated with increased suicide risk in children may pose the same danger to adults.

A study in the journal BMC Medicine has analysed unpublished data on paroxetine, a drug that is used to treat millions of depressed people in Britain every year. Patients taking paroxetine are more likely to attempt suicide than those taking a placebo, according to researchers based at the University of Oslo in Norway.

Paroxetine, which is sold as Seroxat and Paxil, belongs to a class of drugs called selective serotonin re-uptake inhibitors (SSRIs). These drugs increase the availability in the brain of serotonin, a chemical linked to mood and emotions.

In October 2004, the US Food and Drug Administration insisted that labels carry warnings about the use of paroxetine in children. This followed analysis of clinical trials that showed an increase in suicidal thoughts and behaviours in children taking the drug.

Britain's Committee on Safety of Medicines had issued warnings much earlier, in June and September 2003. They advised practitioners that paroxetine should not be used to treat depressive illness in children and adolescents under the age of 18 years. A few months later, in December 2003, the committee extended this warning to cover all SSRIs except fluoxetine (Prozac) for the treatment of depression in children.

But both the UK and US agencies concluded that the data did not confirm an increased risk in adults. They said that the benefits of taking these drugs outweighed the risks for depressed people.

In February 2005, researchers published studies on drug-company data that were submitted for safety review to the London-based Medicines and Healthcare products Regulatory Agency. They found no evidence that SSRIs increase the risk of suicide in adults, but warned doctors that such dangers could not be ruled out (D. Gunnell, J Saperia and D. Ashby Br. Med. J. 330, 385-388).

Their analysis of paroxetine was incomplete because the data supplied by the manufacturer, GlaxoSmithKline, did not allow researchers to distinguish between suicidal thoughts and suicide attempts.

Ivar Aursnes and his colleagues applied to

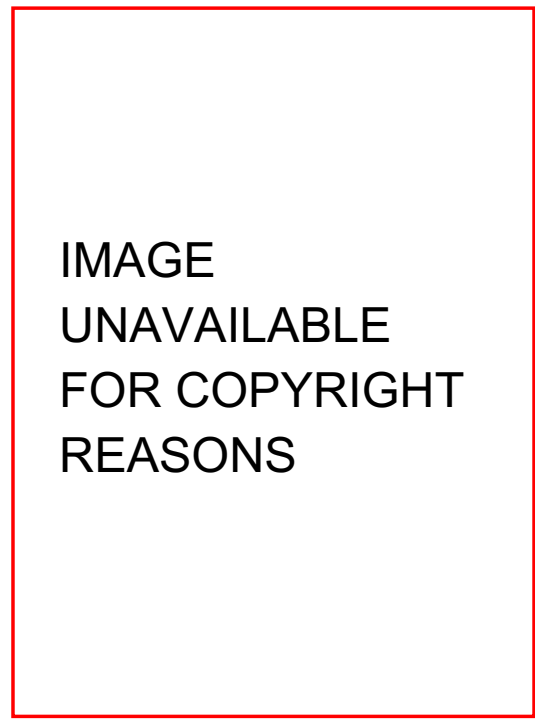

The drug paroxetine may exacerbate suicidal thoughts in adults as well as children.

the Norwegian Medicines Agency to obtain the unpublished data on paroxetine. After a year of correspondence, they were finally granted access to the original drug-company data. Aursnes and his team looked at 16 studies of depressed adults who had been randomly assigned paroxetine or a placebo.

The researchers compared the number of suicides and suicide attempts in the 916 patients taking paroxetine with that in 550 taking the placebo. Eight patients attempted suicide - seven of them were taking paroxetine. The probability that the drug increased the risk of suicide was $90 \%$ in the participants that were studied.

"The use of these drugs needs to be more restricted," Aursnes told Nature. "People should be made aware of the dangers."

Doctors also prescribe paroxetine for problems such as generalized anxiety disorder and social anxiety. "But the risk of suicide is specific to patients receiving paroxetine for depression," says Aursnes, because such people may already have suicidal tendencies.

Tim Kendall, co-director of Britain's National Collaborating Centre for Mental Health, says that the results need to be compared with previously published studies. "We need to look at this more carefully," he says. Jennifer Wild 